\title{
Erratum to: Globalization and Change in Higher Education
}

\section{Erratum to: \\ B. Barrett, Globalization and Change in Higher Education, https://doi.org/10.1007/978-3-319-52368-2}

The original version of the book was inadvertently published with some errors in Appendix, Appendix reference and Index, which have been now corrected. 